\title{
Severity of Depression and Magnitude of Productivity Loss
}

Arne Beck, $P b D^{1,2}$

A. Lauren Crain, $P b D^{3}$

Leif I. Solberg, $M D^{3}$

Jürgen Unützer, $M D, M P H^{4}$

Russell E. Glasgow, $\mathrm{PbD}^{5}$

Michael V. Maciosek, $P b D^{3}$

Robin Whitebird, $\mathrm{PbD}, \mathrm{MS} W^{3}$

${ }^{1}$ Kaiser Permanente, Denver, Colorado

${ }^{2}$ Department of Family Medicine, Univer sity of Colorado Denver Health Sciences Center, Denver, Colorado

${ }^{3}$ HealthPartners Research Foundation, Minneapolis, Minnesota

${ }^{4}$ University of Washington, Seattle, Washington

${ }^{5}$ National Cancer Institute, Washington, DC

\begin{abstract}
PURPOSE Depression is associated with lowered work functioning, including absences, impaired productivity, and decreased job retention. Few studies have examined depression symptoms across a continuum of severity in relationship to the magnitude of work impairment in a large and heterogeneous patient population, however. We assessed the relationship between depression symptom severity and productivity loss among patients initiating treatment for depression.
\end{abstract}

METHODS Data were obtained from patients participating in the DIAMOND (Depression Improvement Across Minnesota: Offering a New Direction) initiative, a statewide quality improvement collaborative to provide enhanced depression care. Patients newly started on antidepressants were surveyed with the Patient Health Questionnaire 9-item screen (PHQ-9), a measure of depression symptom severity; the Work Productivity and Activity Impairment (WPAI) questionnaire, a measure of loss in productivity; and items on health status and demographics.

RESULTS We analyzed data from the 771 patients who reported being currently employed. General linear models adjusting for demographics and health status showed a significant linear, monotonic relationship between depression symptom severity and productivity loss: with every 1-point increase in PHQ-9 score, patients experienced an additional mean productivity loss of $1.65 \%(P<.001)$. Even minor levels of depression symptoms were associated with decrements in work function. Full-time vs part-time employment status and self-reported fair or poor health vs excellent, very good, or good health were also associated with a loss of productivity $(P<.001$ and $P=.045$, respectively).

CONCLUSIONS This study shows a relationship between the severity of depression symptoms and work function, and suggests that even minor levels of depression are associated with a loss of productivity. Employers may find it beneficial to invest in effective treatments for depressed employees across the continuum of depression severity.

Ann Fam Med 2011;9:305-311. doi:10.1370/afm.1260.

\section{INTRODUCTION}

$\mathrm{D}$ epression is prevalent and incurs substantial indirect costs associated with reduced work functioning, including absences, impaired productivity, and even decreased job retention across a wide variety of occupations. ${ }^{1-4}$ In addition, several studies have shown that even minor or subthreshold depression (including dysthymia) is related to poorer work performance. ${ }^{5,6}$ Fewer studies have examined depression symptoms across a continuum of severity in relationship to the magnitude of work loss that includes both absences and impaired productivity. Simon et $\mathrm{al}^{7}$ found that among outpatients treated for bipolar disorder, depression severity was strongly and consistently associated with a decreased probability of employment and more days of absence due to illness. Backenstrass et $\mathrm{al}^{8}$ characterized a spectrum of depressive symptoms across 3 increasing levels of severity (nonspecific, minor, and major) and found an increasing number of days of absence due to illness with each additional level of 
symptom severity. Both of these studies had selected samples, however-patients with a bipolar disorder diagnosis and patients from 6 family practices in a small town near Heidelberg, Germany, respectivelylimiting their generalizability.

The goal of this study was to investigate further the relationship between a continuum of depression symptom severity and the magnitude of productivity loss in a large, heterogeneous, and representative sample of outpatients initiating treatment for depression.

\section{METHODS}

\section{Setting}

We analyzed baseline data collected for a study of a statewide depression quality improvement initiative in Minnesota, called Depression Improvement Across Minnesota: Offering a New Direction (DIAMOND). All groups and clinics that intended to participate by implementing the initiative in their primary care clinics were invited to be in the DIAMOND study. A total of 88 clinics from 23 medical groups participated. Details on the study design and methods are presented elsewhere. ${ }^{9}$

\section{Patient Recruitment and Enrollment}

All patients with health plan claims data showing them to be newly started on antidepressant medications at a participating clinic were identified on a weekly basis by the health plans and sent a letter about the study, providing them a 1 -week opportunity to opt out before being called by the research survey center to determine eligibility for participation and to complete a baseline questionnaire by phone. Patients were eligible if they were older than 18 years of age, had filled a new antidepressant prescription (and none in the prior 4 months) from a primary care clinician at a participating clinic for the treatment of depression, and had a depression symptom severity score of 7 or higher on the Patient Health Questionnaire 9-item screen (PHQ-9). Although part- or full-time employment was not an eligibility criterion for participation in the larger DIAMOND study, because we focused on the relationship between productivity loss at work and depression, we included in our analysis only the subset of patients employed for wages at least part time. Patients completing this baseline questionnaire were also asked for permission to resurvey them 6 months later.

The study protocol was reviewed, approved, and monitored by the HealthPartners Institutional Review Board.

\section{Measures}

We used patient self-report questionnaires to obtain information on depression severity, work absence and productivity impairment, health status (a single item asking patients to rate their overall health), and demographic characteristics. The PHQ-9 was used to measure the severity of depression symptoms. It is widely accepted as a valid measure of depression severity. ${ }^{10-13}$ Questions about work function were obtained from the Work Productivity and Activity Impairment (WPAI) questionnaire, ${ }^{14-16}$ a self-report measure of the amount of absence from work due to health problems, as well as productivity impairment while at work ("presenteeism") experienced during the previous 7 days. We computed additional measures to estimate the percent of work time missed due to health, percent impairment while working due to health, and percent of overall productivity loss due to health. ${ }^{16}$ Percent work time missed due to health, a measure of absenteeism, was calculated as the hours missed during the previous 7 days divided by the hours missed plus the hours worked during this period. Percent impairment while working due to health, a measure of presenteeism, was calculated as a 10 -point rating of degree of impairment while at work divided by 10 . The number of hours of productivity impairment at work was calculated as the hours actually worked multiplied by the percent impairment while at work. The proportion of expected work time that was missed or affected because of health problems over the previous 7 days (productivity loss) was calculated as the percent of work time missed plus the percent of time at work multiplied by impairment while there.

\section{Statistical Analyses}

To investigate the relationship between depression symptoms and productivity loss while adjusting for potential confounders, we estimated general linear models, using the PROC GLM program of SAS, version 9.1.3 (SAS Institute Inc, Cary, North Carolina). This analytic approach was chosen after determining that there were no significant clustering effects and, therefore, the GLM is equivalent to a mixed model. Because the relationships between depression and both work loss and productivity were similar, we used the combined variable, productivity loss, in our subsequent multivariate modeling. The PHQ-9 scores and productivity loss were both treated as continuous variables in the model. We included as covariates multiple demographic variables - age, sex, race/ethnicity (non-Hispanic white vs Hispanic or nonwhite), education (high school or less vs some college or more), employment status (full vs part time), and marital status (coupled vs single) — and self-reported health status, categorized as a combination of excellent, very good, and good vs a combination of fair and poor. We tested for interaction terms between PHQ-9 and covariates and did not find any to be significant, so we eliminated the interaction terms from the model. 


\section{RESULTS}

\section{Patient Enrollment and Demographic Characteristics}

During a 25-month period, 11,889 patient names were submitted to the research survey center; however, $40 \%$ of these patients could not be contacted, and of those who agreed to be screened, $75 \%$ did not meet eligibility criteria for the study. The study enrollment data are shown in Table 1, indicating that to date, 1,168 patients have been contacted, assessed for eligibility, consented, and enrolled. We analyzed data on the relationship between depression and work impairment for the subsample of 771 patients $(66 \%)$ who reported that they were working for wages either full or part time at the time of their interview. Demographic characteristics of these patients are shown in Table 2.

\section{Depression Symptoms}

We divided PHQ-9 scores into 4 ordinal categories corresponding to increasing levels of depression severity. A score of 7 to 9 indicates mild or minor symptoms of depression, a score of 10 to 14 is in the moderate depression range, 15 to 19 is consistent with major depression and considered a diagnostic threshold, and 20 or higher is considered severe depression. The plurality of patients (292 or $38 \%$ ) had scores in the moderate range of depression symptoms, followed by scores in the minor range (263 or $34 \%)$, major range (159 or $21 \%)$, and severe range (57 or $7 \%)$. The mean PHQ-9 score was $12.2(\mathrm{SD}=4.3)$.

\section{Work Loss and Productivity}

Table 3 presents descriptive data on the WPAI items. Patients reported that over the previous 7 days, an average of 3.1 hours, or $8.0 \%$, of their total usual working hours were missed because of health conditions. The average rating of the degree of impairment while at work was 3.5 on
Table 2. Demographic Characteristics of Enrolled Patients Working for Pay $(\mathrm{N}=771)$

\begin{tabular}{|c|c|c|c|}
\hline Characteristic & $\begin{array}{c}\% \text { or } \\
\text { Mean (SD) }\end{array}$ & Characteristic & $\begin{array}{c}\% \text { or } \\
\text { Mean (SD) }\end{array}$ \\
\hline Female & 74.8 & Employment & \\
\hline Age, y & $42.5(12.4)$ & Employed for wages & 90.1 \\
\hline Ethnicity & & Self-employed & 8.0 \\
\hline Hispanic & 3.0 & Student & 1.0 \\
\hline Non-Hispanic white & 90.2 & On disability & 0.8 \\
\hline Race & & Marital status & \\
\hline American Indian & 0.5 & Married & 52.8 \\
\hline Asian & 0.8 & Divorced & 15.8 \\
\hline Black, African American & 3.8 & Separated & 3.2 \\
\hline Multiracial & 1.4 & Unmarried couple & 8.4 \\
\hline Native Hawaiian, & 0.4 & Widowed & 1.7 \\
\hline Alaska Native & & Never married & 18.0 \\
\hline Other & 1.6 & Functional health status & \\
\hline Unknown & 0.3 & Excellent & 7.0 \\
\hline White & 91.3 & Very good & 29.6 \\
\hline Education, highest level & & Good & 40.6 \\
\hline Grade 1-11 & 3.9 & Fair & 18.5 \\
\hline High school & 21.4 & Poor & 4.3 \\
\hline Some college & 39.3 & & \\
\hline College graduate & 24.0 & & \\
\hline Graduate degree & 11.4 & & \\
\hline
\end{tabular}

\section{Table 3. WPAI Items and Related Measures}

Item or Measure (No. of Patients Responding)

Mean (SD)

During the past 7 days, how many hours did you miss from work because of your health problems? (740)

During the past 7 days, how many hours did you actually work? (740)

$3.1(8.0)$

During the past 7 days, how much did your health problems affect your pro-

ductivity while you were working?a (721)

Thinking of your regular job, how many days in the past 7 days were you limited in the amount of work you could do, accomplished less than you would

like, or days you could not do your work as carefully as usual? (737)

Percent of work time missed due to health (absenteeism) (740)

$33.8(15.5)$

$3.5(2.6)$

$2.2(2.3)$

Percent impairment at work due to health (presenteeism) (720)

$8.2(20.5)$

$35.2(26.4)$

Percent of work missed or work time impaired due to health (absenteeism or presenteeism) (719)

Hours of impairment at work due to health (720)

$37.8(27.5)$

$12.1(11.0)$

Hours of work missed or work impaired (productivity loss) due to health (719)

$14.2(12.6)$

WPAI $=$ Work Productivity and Activity Impairment questionnaire

a On a 10-point scale, where higher values indicate greater effect. 
the 10 -point scale, representing $35.2 \%$ of total hours worked, or 12.1 hours of productivity affected while at work. The proportion of expected work time that was missed or affected because of health problems over the previous 7 days (productivity loss) represented an average of $37.8 \%$ of employees' usual work hours, or 14.2 hours of work missed or work time impaired because of health. Note that the value of productivity loss as calculated (and described in the Methods section) is not the sum of absenteeism plus presenteeism, because the latter includes only hours actually at work.

\section{Relationship Between PHQ-9 and WPAI}

Figure 1 graphically shows the relationship between each category of depression symptom severity, as assessed with the PHQ-9, and productivity loss. It illustrates the strong linear relationship between depression symptom severity and the combination of work loss and productivity impairment. It also shows that even minor symptoms were associated with a considerable loss of productivity.

The overall multivariate model for productivity loss containing all covariates was significant, and the included variables explained $10.5 \%$ of the variability among patients in this outcome $\left(\mathrm{F}=10.26, P<.001\right.$, model $\left.R^{2}=.105\right)$.

Table 4 displays the individual variables in the model. There was a positive and significant association between PHQ-9 scores and productivity loss, with each 1 -point increase in score associated with an additional $1.65 \%$ loss of productivity $(P<.001)$. In addition, full-time vs parttime employment status and fair or poor health vs excellent, very good, or good health were also associated with greater productivity loss $(P<.001$ and $P=.045$, respectively).

Because our sample of 771 employed individuals represented only $66 \%$ of those with complete baseline data for this analysis, we conducted a subanalysis to determine whether employment status was related to depression severity among all working-age participants, defined as those aged 18 to 64 years. Results indicated that mean PHQ-9 scores were higher among the 325 study participants reporting no employment
Figure 1. Productivity loss (absenteeism and presenteeism combined) by PHQ-9 score at enrollment: percent of work time missed or impairment at work in past 7 days.

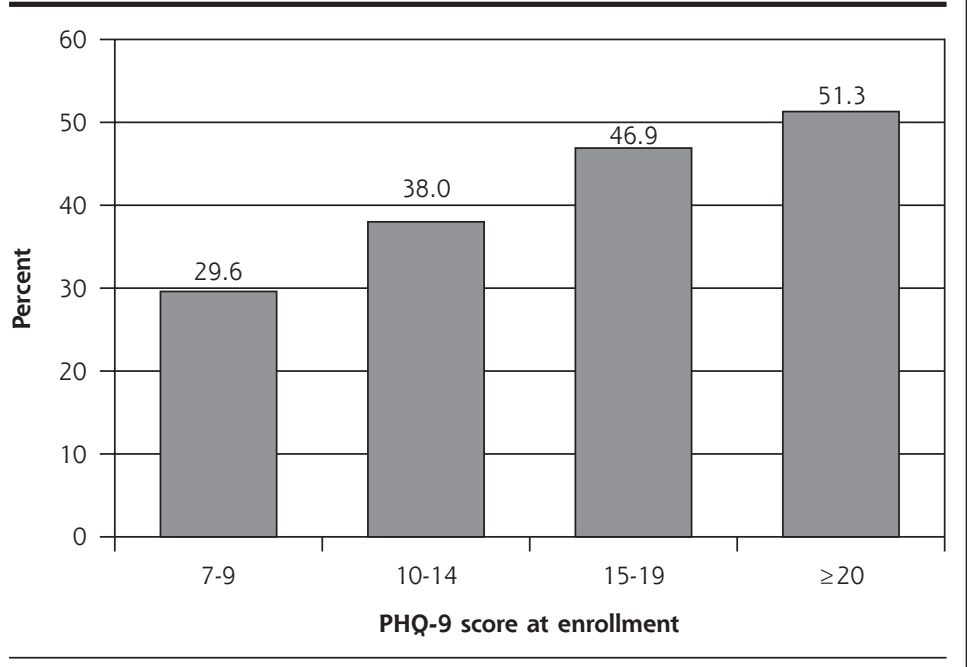

PHQ-9 = Patient Health Questionnaire 9-item screen.

Note: For comparison, the norm for productivity loss for individuals without depression or other chronic conditions is $8.0 \%$.

Table 4. Relationship of Depression Severity (PHQ-9 Score), Demographics, and Health Status to Productivity Loss

\begin{tabular}{|c|c|c|c|c|}
\hline Parameter & $\begin{array}{c}\beta \\
\text { Coefficient }\end{array}$ & Error & $t$ Value & $P$ Value \\
\hline PHQ-9 score ${ }^{\mathrm{a}}$ & 1.65 & 0.24 & 6.98 & $<.001$ \\
\hline Age & 0.006 & 0.08 & 0.07 & .94 \\
\hline Sex (male) & 1.89 & 2.32 & 0.82 & .41 \\
\hline Race/ethnicity (minority ${ }^{b}$ ) & 3.36 & 3.30 & 1.02 & .31 \\
\hline Health (fair/poor) & 4.80 & 2.39 & 2.01 & .045 \\
\hline Education (high school or less) & -1.44 & 2.30 & -0.62 & .53 \\
\hline Employment status (part time) & -9.85 & 2.60 & -3.79 & $<.001$ \\
\hline Marital status (not coupleds) & 3.73 & 2.06 & 1.81 & .07 \\
\hline
\end{tabular}

PHQ-9 = Patient Health Questionnaire 9-item screen.

Notes: The model had an estimated intercept of 36.50 and an error of 1.74. Positive estimates indicate loss of productivity; negative estimates indicate gain of productivity. Productivity loss is defined as the combination of absenteeism (percent of time missed in the past 7 days due to health) and presenteeism (percent impairment at work in the past 7 days due to health). These measures were obtained from the Work Productivity and Activity Impairment (WPAI) questionnaire.

a The general linear model shows the relationship between PHQ-9 score and productivity loss adjusted for all other variables listed in the table.

Combination of Hispanic ethnicity and non-white race categories listed in Table 2.

Divorced, separated, widowed, or never married. (mean $=13.30, \mathrm{SD}=4.90)$ than among the 757 study participants reporting full- or part-time employment $($ mean $=12.17, \mathrm{SD}=4.31)(t=3.62, d f=549.2, P<.001)$.

\section{DISCUSSION}

Baseline data from this large sample of patients demonstrate a linear, monotonic relationship between depression symptom severity and productivity loss; 
that is, the more severe depression symptoms were, the greater the amount of productivity lost. Specifically, we found that for every 1 -point increase in patients' PHQ-9 scores, they experienced an additional mean productivity loss of $1.65 \%$. This relationship was observed after adjusting for, and was not modified by, demographics and self-reported health status.

The finding of greater productivity loss for those employed full time vs part time may be explained by the fact that full-time employees have less flexibility in their schedules, requiring the use of sick absence days and working while negatively affected by depression symptoms. Greater productivity loss among those reporting fair or poor health is consistent with literature on the impact of health conditions on work function.

Although the relationship between depression symptoms and both work loss and presenteeism appeared similar, the relative impact of each differed. The percent of work loss reported over the last 7 days ranged from 4\% (PHQ-9 scores of 7-9) to $17 \%$ (PHQ-9 scores of $\geq 20$ ), whereas the percent of productivity impairment over the same period ranged from 28\% (PHQ-9 scores of 7-9) to 47\% (PHQ-9 scores of $\geq 20$ ). The greater productivity impairment reported likely reflects the limits on sick days available for employees. It also suggests that in relative terms, presenteeism due to depression may represent a more noteworthy problem than absenteeism for employers.

The magnitude of productivity loss in this sample of patients $(38 \%)$ is large compared with normative data for the WPAI that include individuals without health conditions $(8 \%)$, as well as those with such conditions as diabetes (15\%), asthma (15\%), back pain $(16 \%)$, obesity $(18 \%)$, angina $(20 \%)$, and chronic pain (22\%) (personal communication, Steve Schwartz, Director of Research, Health Media, Inc, Ann Arbor, Michigan; August 25, 2010). The greater productivity loss reported by our patients may be due in part to the fact that the study sample was recruited from outpatient clinics during treatment initiation, when depression symptoms were presumably at a peak and recent work function was most affected. In fact, productivity loss for various health conditions is greater when reported in observational studies or clinical trials involving these patients as compared with populationbased surveys. ${ }^{17,18}$ For example, recent studies using the WPAI with clinic-based patient samples show a $28 \%$ productivity loss associated with severe asthma, $38 \%$ for Crohn disease, and 20\% for allergic rhinitis. ${ }^{19}$ Moreover, similar to our findings for depression, the majority of these studies show the loss increases with the severity of the condition.

The finding of greater depression severity among the $34 \%$ of participants not employed raises the ques- tion of whether and how depression severity may contribute to unemployment. Symptoms of depression (lack of initiative, poor self-esteem, etc) are a major barrier to getting a job, holding a job, or both. The relationship of depression severity and productivity loss we report for only the employed sample may therefore well underestimate the impact of depression on work function and employment status in general.

This study adds to the growing body of literature suggesting the importance of treating depression in order to restore psychosocial function in addition to remit symptoms. ${ }^{5}$ These studies have suggested that even minor depression symptom severity is associated with work impairment, and although work performance improves in proportion to depression symptom remission after treatment, ${ }_{,}^{20,21}$ it remains consistently lower among individuals showing clinical improvement in depression compared with nondepressed control individuals. $^{22}$

Fortunately, high-quality depression treatment has been found to reduce symptoms, to improve work function, and to be cost-effective. ${ }^{23}$ Nonpharmacologic treatments may be of benefit for even minor depression, as demonstrated by the randomized trial of Wang et $\mathrm{al}^{24}$ testing telephonic care management of workers with depression (the benefit from pharmacologic treatment of minor depression is less evident ${ }^{25}$ ).

Considering the strength of the relationship between depression symptoms and work performance, our findings also underscore the potential utility of using the PHQ-9 to provide health professionals with insights not only about depression severity, but also about work function. Primary care clinicians who better appreciate the impairment in work function associated with the continuum of minor to severe symptoms of depression may have an extra incentive to treat patients more intensively and to full remission whenever possible, rather than accepting minor degrees of improvement. Treating to full remission may be particularly important given the problem of clinical inertia that depression treatment often poses, that is, lack of follow-up of and treatment adjustments for patients initiated on antidepressants. Moreover, results from this study indicate that different levels of depression symptom severity can be directly translated into magnitude of work impairment. Using a relatively easy to administer instrument such as the PHQ-9 can serve both to help primary care clinicians assess depression in their patients, and once it is identified, to understand at a more precise level how much work impairment may be associated with different levels of depression symptom severity. Given the importance of work in peoples' lives, clinicians might wish to ask patients with high PHQ-9 scores about how their 
depression is affecting their work function, how their work might be affecting their depression, and how treatment for depression or other interventions might help patients not only feel better, but also function better at work and for that matter, in their lives overall. Focusing the discussion on the functional impact of depression may provide depressed patients who are hesitant to acknowledge or treat their depression additional motivation to engage in treatment.

Taking the perspective of employers, these results provide more tangible evidence of the potential labor costs of even minor depression symptoms and the potential to realize a return on investment from ensuring that their employees who experience a broader range of depression symptom severity than might typically be considered to warrant treatment receive the most effective treatments possible. ${ }^{24,26,27}$

Our study has both strengths and limitations. Our large real-world sample was obtained from members of a majority of health plans (including Medicaid plans) across the state of Minnesota within the context of a natural experiment, using minimal inclusion and exclusion criteria. The patients thus constitute a broadly representative sample of depressed primary care patients from a variety of backgrounds, income levels, and occupational categories. The results, therefore, should be widely generalizable (except regarding race and/or ethnicity, which have limited diversity in this geographic region). In addition, our ability to examine the relationship between the actual level of self-reported depressive symptomatology and the amount of work loss and productivity impairment is more informative than that in many studies that have examined work loss and productivity impairment only among patients who received a diagnosis of depression or who reached a threshold of symptom severity classifying them as having major depression. Our findings do suggest that even subclinical levels of depression are associated with work absence and productivity impairments.

One limitation of this study is the lack of detailed data on other health conditions that might be associated with work loss and productivity impairment. The inclusion of self-reported health status provides a less precise measure of disease burden than actual data about medical comorbidities. Unfortunately, we did not have access to comorbidity data across all health plans participating in the study. Regarding the impact of other psychiatric comorbidities on productivity loss, future studies might benefit from using the recently published My Mood Monitor (M-3) instrument to assess a greater number of mental disorders than is possible with the PHQ-9. ${ }^{28}$

An additional limitation is that our analyses were restricted to patients reporting at least some employment, excluding those not in the labor force (eg, retir- ees), because our focus was on work function. Moreover, this study does not provide data on individuals with PHQ-9 scores of less than 7 (ie, nondepressed individuals). As mentioned earlier, however, normative data on productivity loss for nondepressed individuals with no other chronic medical conditions is $8 \%$, which is considerably lower than our findings of $29.6 \%$ for those with minor depression (PHQ-9 scores of 7-9).

Analysis of the relationship between levels of depression symptoms and broad self-reported measures of general functional status across the larger sample of study patients is beyond the scope of this article, but will be reported in a subsequent article. Finally, because insufficient numbers of study patients have reached the follow-up assessment time frame, data are not available to examine the relationship between potential improvements in depression symptoms and work performance, and whether this relationship differs depending on the level of initial depression symptom severity reported. We look forward to reporting these results when such data become available.

In conclusion, this study shows a relationship between levels of depression symptoms and productivity loss, suggesting that even minor levels of depression are associated with decrements in work function. The significant relationship between depression symptoms, as measured by the widely used PHQ-9, and impairments in work function raises the possibility of using this questionnaire as a tool to assess both depression symptoms and work function in patients. Taking the perspective of employers, promoting evidence-based depression management programs to employees experiencing even minor depression may have the potential to reduce work loss and productivity impairment, thus yielding a return on investment in such programs.

Ultimately, the goal of this work is to understand how effective depression care can improve both depression symptoms and work function. Once sufficient numbers of patients have complete 6 -month follow-up data, we also will be able to explore the relationship between depression symptom remission and improvement in work function.

To read or post commentaries in response to this article, see it online at http://www.annfammed.org/cgi/content/full/9/4/305.

Key words: Depression, severity; work impairment; primary care; practice-based research

Submitted November 19, 2010; submitted, revised, February 2, 2011; accepted March 2, 2011.

Funding support: This research was funded by grant 5R01MH080692 from the National Institute of Mental Health.

Acknowledgments: This research would not have been possible without the active support of multiple payers (Blue Cross and Blue Shield of 
Minnesota, First Plan, HealthPartners, Medica, Minnesota Department of Human Services, Preferred One, and U (are), as well as the 24 medical groups who provided information to connect patients to specific study clinics and cooperated with evaluation of patients reporting suicidal thoughts on the PHQ-9. Patient recruitment and surveys have been sustained by Colleen King and her wonderful staff at the HPRF Data Collection Center.

\section{References}

1. Berndt ER, Finkelstein SN, Greenberg PE, et al. Workplace performance effects from chronic depression and its treatment. J Health Econ. 1998;17(5):511-535

2. Kessler RC, Barber C, Birnbaum HG, et al. Depression in the workplace: effects on short-term disability. Health Aff (Millwood). 1999; 18(5):163-171.

3. Lerner D, Adler DA, Chang $H$, et al. Unemployment, job retention, and productivity loss among employees with depression. Psychiatr Serv. 2004;55(12):1371-1378.

4. Lerner D, Adler DA, Chang $\mathrm{H}$, et al. The clinical and occupational correlates of work productivity loss among employed patients with depression. J Occup Environ Med. 2004;46(6 Suppl):S46-S55.

5. Katon W. The impact of depression on workplace functioning and disability costs. Am J Manag Care. 2009;15(11 Suppl):S322-S327.

6. Martin JK, Blum TC, Beach SR, Roman PM. Subclinical depression and performance at work. Soc Psychiatry Psychiatr Epidemiol 1996;31(1):3-9.

7. Simon GE, Ludman EJ, Unützer J, Operskalski BH, Bauer MS. Severity of mood symptoms and work productivity in people treated for bipolar disorder. Bipolar Disord. 2008;10(6):718-725.

8. Backenstrass M, Frank A, Joest K, Hingmann S, Mundt C, Kronmüller KT. A comparative study of nonspecific depressive symptoms and minor depression regarding functional impairment and associated characteristics in primary care. Compr Psychiatry. 2006;47(1):35-41.

9. Solberg LI, Glasgow RE, Unützer J, et al. Partnership research: a practical trial design for evaluation of a natural experiment to improve depression care. Med Care. 2010;48(7):576-582.

10. Kroenke K, Spitzer RL, Williams JB. The PHQ-9: validity of a brief depression severity measure. J Gen Intern Med. 2001;16(9):606-613.

11. Kroenke K, Spitzer RL. The PHQ-9: a new depression and diagnostic severity measure. Psychiatr Ann. 2002;32(9):509-521.

12. Martin A, Rief W, Klaiberg A, Braehler E. Validity of the Brief Patient Health Questionnaire Mood Scale (PHQ-9) in the general population. Gen Hosp Psychiatry. 2006;28(1):71-77.

13. Gilbody S, Richards D, Brealey S, Hewitt C. Screening for depression in medical settings with the Patient Health Questionnaire (PHQ): a diagnostic meta-analysis. J Gen Intern Med. 2007;22(11):1596-1602.
14. Reilly MC, Zbrozek AS, Dukes EM. The validity and reproducibility of a work productivity and activity impairment instrument. Pharmacoeconomics. 1993;4(5):353-365.

15. Mattke S, Balakrishnan A, Bergamo G, Newberry SJ. A review of methods to measure health-related productivity loss. Am J Manag Care. 2007;13(4):211-217.

16. Reilly Associates. Health Outcomes Research. http://www reillyassociates net. Accessed Nov 8, 2010.

17. Bolge SC, Balkrishnan R, Kannan H, Seal B, Drake CL. Burden associated with chronic sleep maintenance insomnia characterized by nighttime awakenings among women with menopausal symptoms. Menopause. 2010;17(1):80-86

18. Erickson SR, Guthrie S, Vanetten-Lee M, et al. Severity of anxiety and work-related outcomes of patients with anxiety disorders. Depress Anxiety. 2009;26(12):1165-1171.

19. Chen H, Blanc PD, Hayden ML, Bleecker ER, Chawla A, Lee JH; TENOR Study Group. Assessing productivity loss and activity impairment in severe or difficult-to-treat asthma. Value Health. 2008;11(2):231-239.

20. Aikens JE, Kroenke K, Nease DE Jr, Klinkman MS, Sen A. Trajectories of improvement for six depression-related outcomes. Gen Hosp Psychiatry. 2008;30(1):26-31.

21. Buist-Bouwman MA, Ormel J, de Graaf R, et al; ESEMeD/MHEDEA 2000 investigators. Mediators of the association between depression and role functioning. Acta Psychiatr Scand. 2008;118(6):451-458.

22. Adler DA, McLaughlin TJ, Rogers WH, Chang H, Lapitsky L, Lerner D. Job performance deficits due to depression. Am J Psychiatry. 2006;163(9):1569-1576.

23. Wang PS, Patrick A, Avorn J, et al. The costs and benefits of enhanced depression care to employers. Arch Gen Psychiatry. 2006;63(12):1345-1353.

24. Wang PS, Simon GE, Avorn J, et al. Telephone screening, outreach, and care management for depressed workers and impact on clinical and work productivity outcomes: a randomized controlled trial. JAMA. 2007;298(12):1401-1411.

25. Fournier JC, DeRubeis RJ, Hollon SD, et al. Antidepressant drug effects and depression severity: a patient-level meta-analysis. JAMA. 2010;303(1):47-53.

26. Wells KB, Sherbourne C, Schoenbaum M, et al. Impact of disseminating quality improvement programs for depression in managed primary care: a randomized controlled trial. JAMA. 2000;283(2): 212-220.

27. Bush T, Rutter $C$, Simon $G$, et al. Who benefits from more structured depression treatment? Int J Psychiatry Med. 2004;34(3):247-258.

28. Gaynes BN, DeVeaugh-Geiss J, Weir S, et al. Feasibility and diagnostic validity of the M-3 checklist: a brief, self-rated screen for depressive, bipolar, anxiety, and post-traumatic stress disorders in primary care. Ann Fam Med. 2010;8(2):160-169. 